P68 (continued)

difficulty for age, (4) time, (5) confidence in teaching lessons, (6) cultural appropriateness, and (7) strengths of curriculum in promoting STEAM education and innovation. Reviewers agreed that the lessons were accurate, incorporated STEAM concepts, and were culturally appropriate for this population. All 9 experts contacted completed the survey and interview. They all agreed that they would be confident in delivering the curriculum themselves.

Conclusion: An expert content review by a diversified panel as part of a formative evaluation process is an effective way to develop an engaging, age-appropriate curriculum.

Funding: USDA.

\section{P69 Adapting a Nutrition Education Program Targeting School-Age Children from In-Person to Virtual During COVID-19}

ClaireWilt, MS, clairewilt4@gmail.com, University of Dayton, 300 College Park, Dayton, OH, 45469; Diana Cuy Castellanos, PhD, RDN, University of Dayton; Elizabeth Freeze, MS, East End Community Center

Background: School-based nutrition education and gardening programs are found to positively affect nutrition knowledge and healthy eating behaviors in children. Due to the limit on in-person learning during the COVID-19 pandemic, innovative technology approaches for continued nutrition and garden education efforts was imperative.

Objective: Therefore, the purpose of this research study was to examine the effect of a virtual nutrition education and gardening program on the identification, intake and likability of fruits and vegetables. Study Design, Setting,

Participants: This was a quasi-experimental study. A total of 14 students (7 Latinx and 7 Non-Latinx White) ages 7-12 years old participated in an all virtual, 6-week summer nutrition education and gardening course.

Measurable Outcomes/Analysis: At baseline and at the end of the 6-week class, participants completed questionnaires to measure their food identification, intake and likeability. Paired samples $t$ tests were run to examine differences in their pre- and post test scores.

Results: Students improved their overall test scores from the pre- to post test by $7 \%(P=0.04)$. There was no difference in scores for Latinx and Non-Latinx students. On a 01 scale, identification (0.06), intake (0.05) and likability (0.10) scores each increased from pre- to post test although not significantly.

Conclusion: COVID-19, has limited the ability for children to be exposed to in-person nutrition education programs they may receive in schools. An effective use of technology to disseminate nutrition and gardening education to schoolaged children can lead to positive nutrition outcomes.

Funding: None.

\section{P70 Association Between Teacher Fidelity to the WISE Intervention and Early Childhood Health Outcomes}

Megan Gremillion, Louisiana Tech University; Tonya Vandenbrink, PhD, Louisiana Tech University; Dong Zhang, $P h D$, University of Arkansas for Medical Sciences; Taren Swindle, PhD, University of Arkansas for Medical Sciences; Peyton Percle, MA, Louisiana Tech University; Julie Rutledge, PhD, rutledge@latech.edu, Louisiana Tech University, PO Box 3167, Ruston, LA, 71272

Background: Early Childhood Educators' (ECEs) foodrelated behaviors can significantly influence child health outcomes (CHOs). The We Inspire Smart Eating (WISE) intervention program trains ECEs in promoting fruit and vegetable consumption by encouraging children's healthy eating habits. WISE provides a 6-hour training and curriculum for weekly sensory-based lessons with 8 target foods. Objective: This study explores the association between ECE WISE fidelity and CHOs. We hypothesize that higher median fidelity scores across time are associated with improved CHOs.

Study Design, Setting, and Participants: Research assistants (RAs) observed lesson fidelity during lessons 8 times across an academic year. RAs scored ECEs on a 1 ("Not at all") to 4 ("Very much") scale for 3 evidencebased practices: hands-on exposure, mascot use, and role modeling. Child participants were African American $63.4 \%$, Caucasian $28.5 \%$, or other $8.1 \%(\mathrm{~N}=659$, male $=$ $4.70,50 \%$ female).

Measurable Outcome/Analysis: Median fidelity scores of ECEs' lessons $(\mathrm{N}=30)$ were calculated to convey fidelity performance across time. CHOs include Body Mass Index (BMI) percentile and Resonance Raman Spectroscopy (RRS) scores. Change scores (BMI $\Delta$ and RRS $\Delta$ ) were calculated by subtracting pre-intervention from post-intervention scores.

Results: In total, 463 children had BMI $\Delta$ and 273 had RRS $\Delta$. A linear regression revealed a significant association between fidelity and BMI $\Delta \mathrm{F}(1,462)=5.068, P=0.025(\beta=$ $-1.315)$.

Results: indicate that ECEs with higher fidelity scores had statistically significantly lower BMI $\Delta$ at post-intervention compared to ECEs with lower fidelity scores. Specifically, a 1-point increase in fidelity was associated with a 1.315 decrease in BMI. The association between fidelity and RRS was not statistically significant $\mathrm{F}(1,272)=0.28, P=0.60$. Multilevel analysis showed variance accounted for by nesting in classroom was not significant.

Conclusion: Children in classrooms with ECEs with higher fidelity may benefit more from the WISE intervention as indicated by BMI $\Delta$. Thus, efforts to improve ECEs' adherence to the evidence-based practices of WISE (eg, implementation strategies) may enhance the impact of the intervention.

Funding: NIH Lincoln Health Foundation. 